\title{
Principios y regulaciones que orientan la evaluación de ensayos clínicos patrocinados por la industria farmacéutica en Comités de Ética de Investigación en Panamá \\ Principles and regulations for the evaluation of clinical research funded by the pharmaceutical industry in Ethics Research Committees in Panama
}

\author{
Claude Vergès, ${ }^{*}$ Noemí Farinoni, ${ }^{* *}$ Matilde Rojas ***
}

https://doi.org/10.36105/mye.2021v32n2.01

\section{Resumen}

Se presentan los resultados de una investigación multicéntrica de evaluación de los Comités de Ética de Investigación (CEI) en Panamá, la cual busca mecanismos que ayuden a fortalecer su capacidad para evaluar, desde un enfoque de bioética y derechos humanos, los protocolos de los ensayos clínicos financiados por la industria farmacéutica o por los centros extranjeros de investi-

\footnotetext{
* Estudió medicina en la Universidad de la Amistad de los Pueblos. Maestría en ciencias de la salud, con especialidad en neumología pediatría en el Hospital del Niño, Doctor José Renán Esquivel, Universidad de Panamá. Maestría en bioética y derecho en la Universidad de Barcelona. Doctorado en educación en la Universidad Interamericana de Educación a Distancia de Panamá (UNIEDPA). Correo electrónico: cverges2004@yahoo.es https://orcid.org/0000-0002-1372-4046

** Socióloga y doctora en andragogía. Profesora e investigadora en el Centro de Investigaciones de la Facultad de Humanidades, Universidad de Panamá, Panamá. Correo electrónico: upnf2020@gmail.com https://orcid.org/0000-0002-5859-5268 *** Maestría en Farmacia Clínica, Universidad de Panamá. Profesora de Farmacia, Universidad de Panamá. Panamá. Correo electrónico: matilderojas25@gmail.com https://orcid.org/0000-0002-2250-263X

Recepción: 10 de diciembre de 2020. Aceptación: 20 de enero de 2021.
} 
gación, así como monitorear la implementación de los protocolos aprobados. Se revisó documentación suficiente y se hicieron entrevistas a miembros de cuatro CEI. A partir de ahí, se concluye que en Panamá los CEI respetan las normas internacionales de funcionamiento y de revisión de protocolos, y que los problemas encontrados guardan relación con su experiencia, el contexto social y la evolución de los intereses de las compañías farmacéuticas.

Palabras clave: Comité de Bioética de Investigación, ensayos clínicos financiados, investigaciones clínicas.

\section{Introducción}

En octubre de 2016 se reunió un grupo de investigadores bajo el nombre de Grupo de Investigación en Ética y Medicamentos, auspiciado por el Departamento de Bioética de la Universidad El Bosque, Bogotá, Colombia, para consensuar el proyecto de investigación titulado «Los Comités de Ética de Investigación y la protección de los participantes en investigaciones biomédicas que residen en América Latina». Su objetivo era «buscar mecanismos que ayuden a fortalecer la capacidad de los CEI latinoamericanos para evaluar los protocolos de ensayos clínicos financiados por la industria farmacéutica o por centros extranjeros de investigación, y monitorear la implementación de los protocolos aprobados desde un enfoque de bioética y derechos humanos» (1). Fueron ocho los países presentes: Argentina, Brasil, Chile, Colombia, México, Panamá, República Dominicana y Perú. ${ }^{1}$ Este análisis de la situación en Panamá forma parte del proyecto multinacional. ${ }^{2}$

\section{La investigación clínica financiada por las compañías farmacéuticas}

La investigación clínica es un requisito esencial de la investigación biomédica y para la comercialización de nuevos medicamentos, 
Principios y regulaciones que orientan la evaluación de ensayos clínicos...

porque asegura que sean eficaces y seguros para la sociedad (2). Sin embargo, la historia ha revelado numerosos ejemplos de abusos que dieron nacimiento a normas internacionales y nacionales. Entre ellas destacan la Declaración de Helsinki, las recomendaciones de la Organización Panamericana de la Salud (OPS) y de la Organización para la Ciencia, la Educación y la Cultura (UNESCO), que han sistematizado los requisitos para la formación de los CEI, cuya función es la protección de los participantes (hasta ahora llamados «sujetos») en la investigación clínica (3).

El desarrollo de Panamá está basado en su capacidad de comercio y servicios, y en las ventajas fiscales que ofrece para la inversión internacional (4). En este marco, la investigación clínica financiada por las compañías farmacéuticas ha tenido un aumento progresivo desde 1990, reclutando investigadores locales en el cuerpo médico, con una ausencia de leyes nacionales sobre investigación hasta el año 2019, ${ }^{3}$ pero con CBI reconocidos por ley desde el año 2003 (5).

\section{Propósito}

El propósito de este estudio ha sido evaluar los criterios, principios y regulaciones que orientan a los miembros de los CBI en la valoración de las investigaciones clínicas farmacológicas y/o farmacogenéticas, patrocinadas por la industria farmacéutica en Panamá.

\section{Método}

En el marco del Encuentro Nacional de Ética de la Investigación en la Universidad El Bosque, en 2016 (1) se desarrollaron talleres de discusión para la realización de esta propuesta. Se trata de una investigación cualitativa y se utilizaron los siguientes instrumentos: un formulario de autoevaluación, revisión de los documentos ofi- 
ciales de los CBI, su información en las páginas web y entrevistas semiestructuradas que fueron probadas previamente a su aplicación.

Al momento de iniciar la investigación en Panamá había ocho CBI acreditados por el Comité Nacional de Bioética (CNBI). Tres comités fueron eliminados porque no evaluaban ensayos clínicos, y otro más por estar en reorganización en esos momentos. Se enviaron cartas de invitación a participar a los presidentes de cuatro comités que evalúan ensayos clínicos financiados: tres del sector público de salud ${ }^{4}$ y uno del sector privado, y a los directores de las tres instituciones públicas para la aceptación de la realización del estudio. Se agregó al CNBI, por la relevancia de su función reguladora y de supervisión de los CBI en Panamá.

Se presentó la documentación necesaria para obtener el registro de la investigación en la Dirección General del Ministerio de Salud y para el aval del Comité de Bioética de la Caja de Seguro Social (que no revisaba estudios clínicos financiados), el cual se obtuvo en octubre de 2017. Durante el 2017 se recolectó el máximo de datos y se presentaron los primeros resultados en un segundo taller multicéntrico en el 2018. Durante el 2018-2019 se realizó el estudio de campo: recopilación de más documentación, de los formularios de autoevaluación hechos a los CBI participantes y 13 entrevistas en los comités seleccionados.

Los entrevistados fueron elegidos según los criterios de selección establecidos. Las entrevistas cumplieron con los criterios de privacidad, consentimiento y confidencialidad; fueron grabadas, transcritas, revisadas, corregidas y codificadas. El registro, procesamiento y análisis de los datos recogidos siguieron los pasos acordados en el protocolo general multinacional.

Los coordinadores del proyecto multinacional revisaron todos los resultados recopilados y completaron la información proporcionada en un segundo encuentro en mayo de 2019. ${ }^{5}$ Toda la documentación original y las grabaciones se guardarán durante cinco años después de la publicación del análisis multinacional y luego serán destruidas. 
Principios y regulaciones que orientan la evaluación de ensayos clínicos...

\section{Resultados}

\section{a) Evolución histórica y marco legal de los CBI}

La creación de los CBI en Panamá ha estado ligada al desarrollo de la investigación clínica financiada y ha seguido los lineamientos de la Organización Mundial de la Salud, a través de la asesoría y supervisión del Programa Regional de Bioética de la OPS (6). Aunque pertenecen a una institución pública o privada que les asegura los recursos necesarios para su funcionamiento, son autónomos en el reclutamiento de sus miembros y en sus decisiones sobre los protocolos sometidos a revisión.

Antes de 1990, la investigación clínica en el Hospital del Niño, Dr. José Renán Esquivel (HDNDJRE), era esencialmente de tipo descriptivo, y realizada por médicos residentes y especialistas en el marco de la docencia médica. Con el cambio de dirección, llegan las primeras solicitudes de investigación clínica financiada. El primer CBI se instaló mediante el memorando DM-M-0041 del 25 de enero de 1991, reemplazando a la Comisión de Investigación existente, y decía: «su función principal es la de proteger el bienestar y los derechos de las personas que participan como sujetos en una investigación» (7).

El Instituto Conmemorativo Gorgas de Estudios de la Salud (ICGES) es el pionero de la investigación en salud en Panamá, y está adscrito al Ministerio de Salud (MINSA). Este rol de líder de la investigación en salud fue formalizado en la Resolución $\mathrm{N}^{\circ} 201$, del 6 de agosto de 1999 (Gaceta Oficial-GO 23,872 del 26 de julio de 1999), en la que se establecieron las políticas, prioridades y normas básicas de investigación en salud, y se atribuyó al CBI del ICGES la responsabilidad de la evaluación ética de los estudios de investigación clínica que se realizasen en las instalaciones y regiones del Sistema Nacional de Salud, así como la interpretación especializada de las pautas recomendadas por los documentos de la OMS y del Consejo de Organizaciones Internacionales de Ciencias Médicas 
(CIOMS). Este Comité se conocía como el Comité Nacional de Bioética del Gorgas. En la Resolución $\mathrm{N}^{\circ} 390$, del 6 de noviembre de 2003 (GO N ${ }^{\circ} 24,938$ del 28 de noviembre de 2003), se adoptaron las Guías Operacionales de Bioética en Investigación. Con la entrada en vigor del Decreto Ejecutivo 1,110, del 6 de junio de 2012 (GO N 27,056-A del 14 de junio de 2012), se realizaron reformas en cuanto a la composición de los miembros del Comité Nacional de Bioética de Investigación, y se trasladó del Instituto Conmemorativo Gorgas a su actual sede en la Secretaría Nacional de Ciencia y Tecnología (SENACYT). Mediante la Resolución 013 del 5 de julio de 2012 de la Junta Directiva del ICGES (GO N² 27,090 del $1^{\circ}$ de agosto de 2012), se creó el Comité de Bioética de Investigación de esta institución, con la finalidad de asegurar la revisión ética, legal y metodológica de todos los protocolos de investigación que fueran sometidos en ese comité (8).

A partir del año 2012, y con el Decreto Ejecutivo 1,110 del 6 de junio de 2012 (GO N 27,056-A del 14 de junio de 2012), se realizaron reformas en cuanto a la composición de los miembros del Comité Nacional de Bioética de Investigación (CNBI), su procedencia y el número de miembros. Se detalló el ámbito de sus funciones para acreditar, supervisar y auditar los Comités de Bioética de Investigación locales e institucionales, y se delimitó, dentro de sus competencias, la evaluación de los protocolos con población indígena y de los protocolos de investigación con células madre. Con el Decreto Ejecutivo 1,317 del 25 de julio de 2012 (GO N² 27,089-A, del 31 de julio de 2012) se volvieron a hacer cambios, tanto en el número como en la naturaleza de la composición del CNBI: se aumentó el número de miembros, de nueve a once (que representan a instituciones gubernamentales, centros de investigación, hospitales, universidades y a la sociedad civil), los cuales son ratificados por el Ministerio de Salud. Entre los años 2011-2014 la imagen del CNBI se vio empañada, debido a conflictos de intereses que pusieron en peligro la seguridad de los pacientes. Los comités institucionales locales trataron de marcar su diferencia, extremando los 
Principios y regulaciones que orientan la evaluación de ensayos clínicos...

requisitos de revisión ética. A raíz de esta situación, el CNBI sufrió nuevos cambios en el Decreto Ejecutivo $\mathrm{N}^{\circ} 1,843$, del 16 de diciembre de 2014. Actualmente está normado mediante la Ley $\mathrm{N}^{\circ}$ 84, del 14 de mayo de 2019. Está adscrito al Despacho Superior del Ministerio de Salud, con independencia y autonomía en sus funciones, y recibe el apoyo logístico de la Secretaría Nacional de Ciencia, Tecnología e Innovación (SENACYT). «Está encargado de orientar las políticas públicas de investigación, garantizar la calidad del sistema de investigación clínica, mediante la acreditación de los Comités de Bioética o de Ética de Investigación institucionales, y evaluar estudios clínicos en células madre» (9). Según la página web del CNBI, las instituciones tanto públicas como privadas pueden solicitar la acreditación de un CBI y, desde 2018 a la fecha de presentación de este informe, se han acreditado cinco nuevos CBI, dos de ellos de universidades privadas.

De acuerdo con su página web, el Hospital Punta Pacífica, actualmente Pacífica Salud, es un hospital privado afiliado al Johns Hopkins Medicine International y cuenta con una alta tecnología. El personal médico y de enfermería cuenta con un programa de educación continua en temas de relevancia, a través de videoconferencias en tiempo real y con simposios de actualización en diversas ramas de la medicina. El hospital desarrolla estudios de investigación de enfermedades. El Comité de Bioética en Investigación (CBI-PP) ha sido reestructurado en 2017 y ha sido recertificado en 2019 (10).

b) Composición de los Comités de Bioética de la Investigación y formación de los miembros

Los CBI de Panamá han tratado de ceñirse a las recomendaciones de la OPS sobre su composición y funcionamiento. La cantidad mínima de miembros es de cinco y el total puede llegar a trece. Las recomendaciones de la UNESCO (2005) sobre la formación y funcionamiento de los CBI insisten en la importancia de la multidisci- 
plinaridad $(11,12,13)$. Sin embargo, los médicos representan entre el 50\% y 75\% de los miembros en los comités estudiados. Los demás miembros son del equipo de salud: enfermeras, farmacéuticos, fisioterapistas, salubristas, trabajadores sociales. Para participar en un CBI se exigen conocimientos científicos en salud y en metodología de la investigación. ${ }^{6}$ Algunos miembros son o han sido investigadores clínicos, lo que les permite aportar su experiencia para la valoración de la factibilidad y pertinencia de algunas modalidades de un protocolo sometido a revisión.

La representación de miembros externos a la institución no pasa de un miembro, a excepción del CBI-ICGES, que cuenta con cuatro miembros externos (25\%), los cuales compensan la presencia de tres directores de departamento de la institución. Estos miembros son seleccionados por invitación, previa recomendación de uno de los miembros del CBI y de organizaciones de apoyo a los pacientes (CBI-PP, entrevistas), o de un organismo de defensa de los intereses de un sector particular, como la Secretaría de la Niñez en el CBI-HDNDRJRE. Los miembros de la comunidad tienen los mismos deberes y derechos que los demás miembros y participan activamente en la revisión de los protocolos, luego de los tres meses de su integración al comité. Este periodo varía según la dinámica de cada comité, de la formación profesional, personalidad y seguridad del miembro externo. Frecuentemente ocurre que sólo evalúan si la forma del consentimiento puede ser entendida por el sujeto de experimentación. Pero no saben si el sujeto ha entendido o no, porque ningún comité ha hablado con los participantes.

Los entrevistados reconocen que la falta de participación de miembros externos es una debilidad, pero la relacionan con su funcionamiento: ad honorem y en horario que puede ser conflictivo para el trabajo de estas personas; por lo tanto, la búsqueda activa de algunos CBI se dirige hacia las profesiones liberales y los jubilados, con un éxito relativo, ya que puede llevar meses encontrar a un(a) voluntario(a). 
Principios y regulaciones que orientan la evaluación de ensayos clínicos...

Hasta el 2016 se aceptaban los cursos de Buenas Prácticas Clínicas (BPC) otorgados por las compañías farmacéuticas, además de los cursos por internet del CITI PROGRAM o del Instituto de Salud de los Estados Unidos (NIH). A partir de 2018, el CNBI estipuló uniformar la preparación de los miembros de los CBI en Panamá y evitar los posibles sesgos y conflictos de intereses, ofreciendo cursos de actualización gratuitos. Los cursos obligatorios son los de Protección del Participante y BPC, impartidos por cualquiera de los comités acreditados y válidos por tres años.

\section{c) Recursos de los Comités de Bioética de la Investigación}

Los CBI públicos deben someterse a las reglas administrativas de este sector. Todas las instituciones deben poner a su disposición una oficina propia y el equipo necesario para responder a las exigencias de sus funciones, así como una secretaría técnica. ${ }^{7}$ Es obligatorio que mantengan toda la información relativa a su funcionamiento en un sitio web propio y de manera actualizada.

Los CBI encuestados cobran por la revisión de los protocolos, según una tarifa publicada en su sitio web (CBI-HDNDRJRE, CBI-ICGES) o informada durante el trámite de solicitud de revisión (CBI$\mathrm{PP})$. En el caso de los CBI públicos, este cobro va al fondo común de la institución, y su uso está sometido a los requisitos de ejecución de actividad. Los CBI privados administran su presupuesto, lo que les permite otorgar una dieta a los miembros para asistir a las reuniones y para revisar un protocolo de investigación. Tanto para los CBI de las instituciones públicas como de las privadas, el pago por revisión no implica la aprobación rápida o automática del protocolo sometido a revisión por el investigador principal. ${ }^{8}$

Los CBI no tienen los recursos para llevar a cabo algunas de las responsabilidades que han adquirido: supervisiones sistemáticas a los centros de investigación y entrevistas con los pacientes para comprobar que realmente han entendido el consentimiento infor- 
mado y que no corren riesgos innecesarios ni comprometen la integridad de la información. Sin embargo, están conscientes de ello y, en 2017 y 2018, tres comités realizaron cuatro supervisiones a centros de investigación.

\section{d) Metodología de trabajo de los Comités de Bioética de la Investigación}

Cuando ingresa un protocolo de investigación clínica financiado por una compañía farmacéutica, es sometido al mismo proceso de registro, distribución a los revisores y programación para la discusión en reunión ordinaria, que las demás investigaciones clínicas no financiadas.

En el CBI-HDNDRJRE, la documentación es distribuida a todos los miembros para su revisión, por la percepción de la particular vulnerabilidad de los participantes (menores de 15 años) y por el volumen de protocolos sometidos a revisión. En todos los demás CBI son designados dos revisores de manera secuencial, y tienen quince días para presentar su informe al pleno en una reunión ordinaria, en la que se discuten los interrogantes.

Cuando los revisores se han puesto de acuerdo previamente y falta uno de manera imprevista, el miembro presente habla en nombre de los dos. Si ambos se ausentan, entonces se reprograma la discusión. Luego de intercambiar consideraciones sobre puntos dudosos o aclarar actualizaciones científicas o éticas, los miembros toman la decisión por unanimidad. Está previsto un sistema de votación en caso de no llegar a un acuerdo, pero no ha sido empleado en ninguno de los comités encuestados.

\section{e) Conflictos de interés}

Todos los CBI han aprobado un Procedimiento Operativo Estándar (POE) para el manejo de los posibles conflictos de interés de sus miembros. Este POE exige el retiro de las discusiones sobre un 
Principios y regulaciones que orientan la evaluación de ensayos clínicos...

protocolo particular cuando el miembro pertenece al equipo investigador de este protocolo, trabaja en el mismo departamento o tiene relaciones con uno de los investigadores. Esta disposición se cumple sin conflictos, porque es del interés del CBI que la investigación no sea objeto de cuestionamiento por parte de la comunidad científica, institucional y en general.

\section{f) Centros de investigación e investigadores principales}

Aparte del ICGES, que es un instituto dedicado únicamente a la investigación básica, clínica y epidemiológica en salud, los demás centros de investigación están en los hospitales públicos como parte de la enseñanza médica o como servicio autónomo y en las clínicas privadas, consultorios individuales o centros dedicados a la investigación. No hay legislación específica sobre los centros de investigación. Desde 2015, el CBI-ICGES ha solicitado a los investigadores de ensayos clínicos un mínimo de requisitos para hacer frente a las posibles urgencias, tales como equipo de reanimación en el lugar de atención de los participantes y estar lo más cerca posible de un cuarto de urgencias.

En los últimos años varios investigadores pertenecientes a instituciones públicas han creado su propio centro de investigación fuera de la institución donde laboran, pero en algunos casos someten el protocolo a revisión en el comité de la institución pública de la que son parte. No hay ningún impedimento legal para ello; sin embargo, este hecho puede representar un dilema ético y un conflicto de interés.

El 95\% de los ensayos clínicos financiados y registrados en los CBI considerados son llevados a cabo por un equipo de tres investigadores o más, en su mayoría médicos especialistas en la patología objeto de estudio. En Panamá, un país de pocos habitantes y con pocos médicos especialistas, se observa una recurrencia de los equipos de manera regular (por ejemplo, Infectología y Oncología). 


\section{g) Registro de la información sobre los ensayos clínicos}

El CBI-HDNDRJRE y el CBI-ICGES publican en su sitio web el informe anual de todas las investigaciones revisadas por ellos desde el año 2008. Siendo el HDNDRJRE un hospital formador de médicos, la mayoría de las investigaciones corresponden a las tesis de grado de los médicos residentes.

A partir del 2015, todos los CBI acreditados en Panamá deben entregar un informe mensual y un informe anual al CNBI, el cual los publica en su sitio web. Este informe contiene: el título del estudio y su número de referencia; las fechas de ingreso para revisión, solicitud de correcciones y aprobación; el patrocinador, nombres de los investigadores principales y su categoría y sitio de estudio.

\section{b) Rol de la Agencia Reguladora}

Hasta el 2015, el rol de la agencia reguladora se había delegado al CNBI. A partir de ese año, el MINSA publicó el Decreto Ejecutivo número 6, del 3 de febrero de $2015,{ }^{9}$ con el que creó el Departamento de Investigación encargado de revisar si las investigaciones afectaban las políticas y los programas de salud del MINSA, e instaló una plataforma de registro en su página web. Además, impulsó la redacción participativa de la Ley 84 sobre investigación en salud durante los años 2018-2019. Ésta tomó en cuenta lo siguiente: la protección de los participantes; las prioridades de las líneas de investigación del MINSA y el nombramiento de un comité asesor en investigación; los roles de regulación y fiscalización del MINSA y del CNBI; la evaluación de los protocolos; la educación sobre ética de investigación del CNBI y de los CBI; los recursos humanos, financieros y físicos para el funcionamiento de los CBI; el financiamiento de las investigaciones y los deberes y derechos de los investigadores (14). 
Principios y regulaciones que orientan la evaluación de ensayos clínicos...

\section{i) Los ensayos clínicos: diseño, fases y tipos de medicamentos}

De los informes de protocolos de investigación revisados por los CBI en este estudio entre 2012 y $2017,,^{10}$ el $90 \%$ de las investigaciones clínicas son de fase III sobre eficacia, dosis y seguridad de los medicamentos estudiados. La mayoría de los protocolos utilizan la metodología de grupos aleatorios, simple o de doble ciego, con criterios de inclusión y exclusión detallados y relacionados con la patología en estudio y con los riesgos esperados.

Estos estudios están diseñados en dos etapas, para que el grupo control reciba el medicamento de estudio luego de recolectar los datos del primer grupo. Los nuevos antibióticos son comparados con un antibiótico de uso estándar en estudios tipo doble ciego. Los medicamentos estudiados fueron los siguientes, en orden decreciente: antibióticos, anti-VRS, anti-VIH, ${ }^{11}$ montelukast, dexametasona inhalada, poloxamero, crema con antibiótico, anticuerpos monoclonales. ${ }^{12}$ Por otra parte, se puede considerar que varios antibióticos estudiados, así como los medicamentos inhalados y ciertos anticuerpos monoclonales, son medicamentos me too.

Algunos miembros de los CBI consideran que el impacto social de los ensayos me too no representa un riesgo mayor, y que no tienen un respaldo legal que les permita no aprobar este tipo de ensayos cuando los demás criterios están cumplidos. Otros consideran que se debe tomar en cuenta el valor social: aumento de precio sin beneficio evidente y carga económica para los pacientes y el sistema sanitario. Esta situación requiere un debate sobre el impacto social: ¿de grupo? ¿de la sociedad entera? Hasta ahora no se ha dado en los CBI, y consideramos que requiere una participación de la sociedad organizada.

Alrededor del 5\% de los estudios son de fase IV de seguimiento del tratamiento de una enfermedad. Estos protocolos surgen de una carencia de los registros oficiales de los ministerios de salud de la región y de la necesidad de diseñar programas para estas enfermedades. A este problema de responsabilidad social del Estado se 
suma la alianza entre una compañía farmacéutica y una asociación médica que comparten los datos personales de los registros (problema de privacidad) y que permitiría el monopolio de estos datos por esta compañía. ${ }^{13}$

Cerca de otro 5\% de los estudios son de fase I, con ensayos de vacunas en pediatría por infectología, de dispositivos intraoculares para glaucoma en oftalmología y de catéteres uretrales en urología.

j) Evaluación de las propuestas de ensayos clinicos

\section{Documentación requerida}

Para revisar un protocolo, los CBI exigen la entrega de una serie de documentos, como son los datos del equipo investigador, de la operatividad de la investigación, del protocolo, las descripciones de los procesos y sobre el medicamento en estudio.

Algunos comités no cuentan con especialista en farmacología, lo que dificulta el análisis del manual del investigador y los riesgos de los participantes. La mayoría de los comités están confrontados a la oposición de las casas farmacéuticas de modificar su protocolo, aduciendo que son multicéntricos y que no es posible hacer cambios para un solo país. ${ }^{14}$

\section{Reclutamiento de los participantes}

Es obligatorio incluir el plan de reclutamiento de los participantes en el protocolo de investigación. Los CBI consideran que los investigadores médicos no pueden reclutar a sus pacientes, sino que deben hacer una publicación comunicando que se va a iniciar una investigación y que las personas se pueden acercar a un miembro del equipo en la dirección indicada. ${ }^{15}$ También el investigador puede solicitar a otros médicos reclutar voluntarios para la investigación. ${ }^{16}$ Estas disposiciones cumplen con el principio de autonomía y transparencia. Sin embargo, surge el interrogante sobre la influencia subjetiva de la relación médico-paciente en el caso de los médicos referentes y de la notoriedad de algunos investigadores. 
Principios y regulaciones que orientan la evaluación de ensayos clínicos...

La selección de los participantes está determinada por su patología y voluntariedad. La mayoría se realiza exclusivamente con participantes de los sectores más pobres y menos educados que dependen de los servicios públicos de salud, apoyándose exclusivamente sobre el factor individual de edad, género o enfermedad, sin tomar en cuenta estos factores externos de vulnerabilidad. ${ }^{17}$ Esta visión «biológica» refleja un desconocimiento de los factores sociales que pueden aumentar los riesgos y que serán importantes al momento de implementarlos en la atención, y para evaluar su impacto en el control de esta patología. Desde el punto de vista bioético, esta práctica choca con la igualdad, la justicia y la no maleficencia (15).

Los incentivos monetarios y de atención a los participantes no están permitidos en Panamá (14), y los CBI son vigilantes del cumplimiento de esta disposición (POE). ${ }^{18}$ Las compañías farmacéuticas pagan a los investigadores para administrar la investigación y esto incluye el reclutamiento de los participantes. En ocasiones se ha escuchado de incentivos a médicos periféricos para que recluten participantes; sin embargo, no hay pruebas oficiales de ello. ${ }^{19}$

\section{Proceso de revisión de los protocolos de ensayos clínicos}

Los pasos para la revisión de un protocolo han sido definidos en un POE y las diferencias entre CBI son mínimas, ya que los indicadores siguen los lineamientos de la OPS adaptados por el CNBI. Actualmente, los POE de todos los CBI consideran que el tiempo máximo de revisión de un protocolo de investigación es de 60 días, pero generalmente se revisa en 30 . El investigador debe contestar a las interrogantes, si las hubiere, en un periodo máximo de tres meses, luego del cual debe someter el protocolo como si fuera por primera vez. La mayoría de los miembros de los CBI entrevistados consideran que este tiempo es suficiente.

\section{Revisión del proceso de consentimiento informado (CI)}

Este paso es considerado como el más importante por todos los comités. Algunos solicitan que el procedimiento para el CI se deta- 
lle en el protocolo, ya que la costumbre es entregar el formulario escrito para que el posible participante lo lea y lo firme. Los POE relacionados detallan los puntos del consentimiento escrito, tanto para los adultos como para los menores de 18 años e incapacitados, según la guía de las BPC. Es obligatorio incluir que hay tratamientos alternativos; definir aleatorización y placebo y la posibilidad de que esté en el grupo que no recibe el tratamiento experimental ${ }^{20}$ indicar cuáles son los beneficios directos e indirectos de esta investigación, los riesgos y las medidas previstas para prevenirlos o mitigarlos. Indicar, además, dónde se realizará la atención de los daños, y debe constar que no tendrán costo para el participante o su familiar. ${ }^{21}$

Los protocolos de investigación biomédica indican que son financiados por la compañía farmacéutica, cuyo nombre figura en la página de encabezamiento, y que los investigadores reciben un pago por su realización. No se indica el monto de este beneficio ni si la institución en la que se desarrolla la investigación recibe también un beneficio económico, porque las leyes actuales no lo exigen. ${ }^{22}$

Los CBI revisan el lenguaje técnico, solicitando aclaraciones y una redacción más comprensible en el marco de la información detallada. Hay que reconocer que los formularios de los CI se han complicado mucho, son demasiado largos (más de 20 páginas) y parecen estar redactados más para evitar problemas legales que para informar a los pacientes. Los CBI solicitan cambios aclaratorios del lenguaje técnico en prácticamente todos los formularios del CI y ajustes al propio proceso en algunos, especialmente para asegurar la confidencialidad y privacidad. Estas solicitudes de cambio son acatadas por los investigadores con el acuerdo de las compañías farmacéuticas financiadoras.

Hace unos cinco años, uno de los CBI confrontó un problema con la solicitud de recolección de lo que quedaba de las muestras biológicas, o de una nueva toma para estudios futuros (broad consent) en el formulario de CI para una investigación. Luego de la revisión de la literatura internacional al respecto y de discusiones 
Principios y regulaciones que orientan la evaluación de ensayos clínicos...

internas, se decidió exigir un formulario aparte, que permita al participante colaborar en la investigación principal, aceptar o rehusarse a entregar sus muestras para investigación futura de manera independiente. Hubo resistencia por parte de la compañía financiadora, pero el comité se mantuvo firme en su decisión y terminaron por aceptar la presentación de dos consentimientos informados independientes.

\section{Eventos Adversos (EA)}

Los CBI han definido cómo y cuándo los investigadores deben reportar los eventos adversos según su nivel de gravedad, así como las desviaciones y las enmiendas. ${ }^{23} \mathrm{El}$ CBI puede solicitar más información sobre un evento particular hasta considerar que se ha resuelto satisfactoriamente. En ocasiones, la entidad financiera patrocinadora ha querido imponer que la información le sea entregada para que ella sea quien informe al CBI (y a las autoridades de salud si compete). Por ello, el aval para la realización de la investigación ha sido condicionado a que la información al CBI sea prioritaria o en paralelo con la entidad financiera patrocinadora.

Otro problema resulta en la atención de los EA que ocurren durante la investigación. Los CBI han estado atentos a que los protocolos especifiquen que los participantes serán compensados por esta atención; sin embargo, algunos protocolos especifican que este monto será «razonable» y que concierne solamente a los eventos adversos «relacionados con el estudio» y no «durante el estudio». Frecuentemente la atención de los EA queda a cargo de las instituciones públicas que brindan la atención necesaria, o del participante que debe utilizar su seguro privado. En Panamá hay dos tarifas: la tarifa del sector público de salud, que es subvencionada por el Estado para que sea un costo mínimo para los usuarios tras una valoración socioeconómica de la familia y de su capacidad de pago. Por su parte, los seguros de salud y los costos de atención en el sector privado han presentado un alza importante en los últimos años. ${ }^{24}$ La vigilancia de los derechos de los participantes y de las 
instituciones auspiciadoras depende de la experiencia y del consenso logrado de cada CBI frente a estos problemas, ya que no existe una legislación específica. Los CBI entrevistados han impuesto el cambio del término «razonable» por el de «compensación según las tarifas oficiales en el país»; esta tarifa puede ser encontrada en los sitios web del Ministerio de Economía y Finanzas y de la Contraloría General de la República.

Otro problema ético resulta de la comprobación de la relación de los EA con los medicamentos del estudio. A menos que sea evidente, lo cual ha sido relativamente raro, esta relación queda a cargo del investigador principal o de la entidad financiera patrocinadora. Ningún miembro de los CBI entrevistados han mencionado algún caso en el cual se establecía esta relación. Tampoco recordaban el uso efectivo del seguro suscrito por la entidad financiera patrocinadora. Estos dos hechos evidencian los límites que tienen los CBI para la verificación de las compensaciones previstas. ${ }^{25}$

\section{Monitoreo y supervisión, integridad de los datos e informes}

Los CBI en Panamá no disponen de un presupuesto para la realización de sus funciones, y esto ha sido un impedimento para una supervisión adecuada. El CNBI solicitó a los CBI acreditados realizar supervisiones cuando recibían interrogantes o quejas de los participantes u observaban inconsistencias en los informes, o lo hacían a solicitud de los propios investigadores. Algunos miembros de los CBI encuestados han participado en estas inspecciones que siguen un procedimiento operativo establecido.

La secretaría técnica del CBI acuerda una fecha de inspección para asegurar la presencia del investigador principal y del responsable administrativo del estudio. El CBI designa una comisión de tres miembros que no tienen conflicto de interés con este sitio o con los investigadores, para que realicen la inspección según un formulario de preguntas y de revisión de documentos. Esta comisión debe tener acceso a todos los documentos de la investigación, respetando el principio de confidencialidad de los datos. El informe 
Principios y regulaciones que orientan la evaluación de ensayos clínicos...

de la inspección con las observaciones es firmado por los miembros de la comisión y los responsables de la investigación al final de la visita, y se presenta para discusión en la siguiente reunión ordinaria del CBI. Ningún CBI ha supervisado la implementación de un ensayo clínico a través de la observación durante la visita de un participante; tampoco existe la capacidad de supervisión de los procesos de reclutamiento y de consentimiento. Esta situación representa un problema que los CBI deben resolver. Todavía hace falta un calendario regular de inspecciones a los sitios de investigación, por la falta de recursos financieros y de transporte, y por las múltiples ocupaciones de los miembros de los CBI, a los cuales no se les reconoce esta carga específica por parte de sus instituciones. La percepción de las autoridades administrativas institucionales en cuanto a la investigación en general, y a la investigación clínica financiada en particular, demanda todavía mayor convencimiento sobre su importancia y, al mismo tiempo, la investigación clínica financiada es percibida como una fuente privada de ingreso.

Durante la implementación de los ensayos clínicos aprobados, el investigador principal debe enviar informes de los avances, según la periodicidad fijada por el CBI que los aprobó. Para los ensayos clínicos de riesgos mínimos este informe es semestral y anual. Para los ensayos con riesgos mayores se puede solicitar informes mensuales o trimestrales, además del informe anual.

\section{Discusión}

El derecho a la vida y a la salud de las personas implica que los medicamentos utilizados sean eficaces, seguros y accesibles. Para ello, los medicamentos puestos a disposición de los enfermos, médicos y farmacéuticos deben cumplir con una serie de etapas de investigación antes de su comercialización, sin desconocer la etapa de farmacovigilancia permanente posterior al consumo (16). La historia de la investigación de medicamentos ha estado plagada de 
violaciones a los derechos humanos de los participantes, las cuales han conducido al establecimiento de leyes, normas y guías internacionales que han servido de modelo para las leyes y normas locales (3). A pesar de estas normas legales, los medios de comunicación reportan escándalos de corrupción en la investigación clínica, que repercuten sobre la seguridad de los participantes y luego en los enfermos (17).

En efecto, la producción de medicamentos es una industria económica importante (18), que encuentra un eco positivo en los decisores económicos de Panamá. La visión económica panameña insiste en las ventajas de su posición geográfica e incentivos fiscales (Resolución de Gabinete no 1, del 7 de enero de 2020). En este marco, la instalación de compañías farmacéuticas en Panamá «hacia la posición de un polo farmacéutico» resulta atractiva, tanto por la facilidad de distribución de medicamentos en la región como por «la agenda de Estado para la atracción de inversión extranjera directa; por las condiciones laborales del país y por la buena receptividad de los gobiernos panameños». ${ }^{26}$ Esta instalación ha sido precedida y acompañada por la realización de ensayos clínicos con medicamentos y vacunas, cuyos resultados han cambiado algunas políticas públicas, ${ }^{27}$ y han consolidado la convicción en los médicos tratantes, investigadores y público general de los beneficios absolutos de la investigación clínica. Sin embargo, es importante considerar la posición institucional y la influencia social de los investigadores ligados a las compañías farmacéuticas para los CBI al momento de tomar decisiones.

Por otra parte, el perfil epidemiológico actual en Panamá es el de una sociedad en transición. En las primeras diez causas de mortalidad figuran los cánceres, las enfermedades cardiovasculares, las enfermedades hematológicas, la diabetes. Las enfermedades infecciosas infantiles y reemergentes también constituyen un problema de salud pública. Frente a esta situación, las autoridades de Salud han apoyado las asociaciones médicas y las guías internacionales (19) ${ }^{28}$ Las investigaciones financiadas de medicamentos entran en 
Principios y regulaciones que orientan la evaluación de ensayos clínicos...

el perfil epidemiológico panameño; el problema ético está en la capacidad del Estado de negociar el acceso de los enfermos a los medicamentos que han resultado beneficiosos. Los CBI han aprobado prolongaciones de investigaciones para beneficio directo de los participantes, pero la ética de equidad y justicia, así como el derecho al acceso a los medicamentos, van más allá de las funciones de estos comités. Otros problemas éticos resultan de los medicamentos me too, y de la presión social y farmacéutica sobre los médicos para utilizarlos como «nuevos», violando los principios de beneficencia/no maleficencia, transparencia y justicia.

En Panamá, el Estado ha delegado en los CBI institucionales el rol de control de la investigación clínica, y en el CNBI su acreditación. Por lo tanto, los criterios y su periodicidad permiten «armonizar las normas científicas y racionalizar los procedimientos documentales y administrativos utilizados en la realización de los ensayos clínicos con medicamentos de uso humano», y se acompañan con una rendición de cuentas de los CBI en cuanto a los protocolos revisados, aceptados y rechazados, además del registro y la publicación en la página web del MINSA. Sin embargo, la diferencia de experiencia entre los CBI de más trayectoria y los nuevos, resalta más la importancia de un acompañamiento inicial y de la asesoría permanente sobre temas poco frecuentes (20). ${ }^{29}$

La recopilación de los documentos de los CBI encuestados y de las entrevistas muestra la conformidad con los lineamientos actuales y la relativa uniformidad de trabajo y proceso de decisiones de estos comités. Cada aspecto de la revisión de un protocolo clínico financiado puede representar un conflicto de intereses entre las diferentes obligaciones: dar respuesta a la investigación y a la empresa financiadora, y asegurar la protección de los participantes y de la sociedad.

La transferencia de conocimientos y de tecnología para el desarrollo de las capacidades locales y nacionales en investigación y atención de salud forma parte del impacto social de la investigación, y representa la expresión de la solidaridad en la cooperación 
internacional plasmada en la DUBDH (UNESCO, 2005). Los exámenes especializados son realizados en un laboratorio fuera del país que está ligado a la compañía farmacéutica, dejando los exámenes comunes para los laboratorios locales. Las normas nacionales e internacionales y los reglamentos de los CBI no mencionan este punto como parte de la revisión que deben realizar; por lo tanto, no se encontraron POE sobre este tópico.

Por otra parte, la mayoría de los protocolos internacionales no presentan el presupuesto de la investigación, invocando su carácter multicéntrico y la confidencialidad de estos datos frente a la competencia del mercado. ${ }^{30}$ Esta situación puede prestarse a prácticas de corrupción institucional y de los investigadores (21), y debe ser objeto de un debate. ${ }^{31}$

Los CBI exigen, como prueba de transparencia, que el formulario de CI indique si el equipo investigador recibe emolumentos, pero no solicitan el monto del mismo ni el monto de la indemnización a la institución que alberga la investigación. Weissman y colaboradores (22) reportan que más de la mitad de los miembros de CBI consideran que las relaciones con la industria farmacéutica afectan la integridad de la investigación. ${ }^{32}$

Estas situaciones se analizaron durante las discusiones para la redacción de la Ley 84 de 2019, que regula la investigación. ${ }^{33}$ Es un paso importante y falta incluir los valores éticos de equidad y justicia entre los participantes en cuanto a la elaboración, análisis y publicación de la investigación. La reglamentación de la ley deberá tener en cuenta estos principios para abordar los aspectos específicos de estos posibles acuerdos. Tanto en el caso de la transferencia de tecnología como en la transparencia del financiamiento y de la gestión de la investigación, los CBI podrían hacer de facilitadores entre investigadores y administradores institucionales en el marco de los principios éticos de responsabilidad social.

El respeto de la dignidad de la persona implica el respeto y la protección de su privacidad y de la confidencialidad de sus datos. LOs CBI han inscrito en sus POE la vigilancia del manejo confidencial 
Principios y regulaciones que orientan la evaluación de ensayos clínicos...

de los datos de los participantes. Las disposiciones para preservar esta confidencialidad (recolección, conservación y destrucción) deben figurar tanto en el protocolo como en el formulario del consentimiento informado; esta obligación no ha tenido cuestionamiento hasta el momento por parte de los patrocinadores financieros. ${ }^{34}$

En cuanto al manejo de las muestras biológicas, los POE requieren que se especifique cómo y cuánto tiempo se guardarán las muestras, quién tendrá acceso a ellas y cómo serán destruidas. Esta misma información debe incluirse en el consentimiento informado. Sin embargo, es frecuente que los CBI deban exigir su inclusión luego de revisar la documentación aportada. La Ley 3 General de Trasplantes de Componentes Anatómicos de 2010 establece las condiciones de importación y exportación de muestras biológicas. A pesar de ello, las compañías farmacéuticas utilizan la posibilidad que da la ley de realizar estudios fuera cuando no existen en Panamá. Últimamente, las compañías condicionan la participación en la investigación de un medicamento al consentimiento para guardar las muestras para investigaciones futuras, lo que representa una coerción para el participante, que tiene la percepción de que esta investigación representa una esperanza para prolongar su vida o para disminuir su sufrimiento.

Los miembros del CBI deben poseer conocimientos de las metodologías de investigación clínica, hasta que los comités de revisión científica sean operativos. Algunos artículos postulan que es responsabilidad de los miembros revisar el manual del investigador sobre el tipo de medicamento estudiado, la metodología elegida y los posibles sesgos $(23,24)$. Sin embargo, estos manuales son cada vez más complicados, y la sensibilidad crítica de los miembros de CBI es muy importante para sospechar posibles lesiones a la seguridad de los participantes. ${ }^{35}$ Es importante que, por lo menos, uno de los miembros del comité sea farmacéutico, y que se pueda solicitar la asesoría de un médico veterinario sobre los tipos de animales utilizados y la interpretación de los resultados en ellos, aplicando la relación riesgo-beneficio, no maleficencia, prudencia y beneficencia. 
La mayoría de los investigadores en estos estudios clínicos son médicos que realizan investigaciones en centros de investigación o en su centro de atención privada y, en ocasiones, pública. Para los participantes es difícil separar la atención de la investigación, a pesar de que todos los documentos mencionan que es una investigación, ya que el médico investigador tiene el título de la especialidad que atiende su enfermedad o que seguirá su tratamiento en el futuro. Aun cuando ha sido reclutado por otro médico, representa un sesgo difícil de resolver por el tamaño de la población panameña y de los médicos especialistas.

La DUBDH, la Declaración de Helsinki y las guías de CIOMS insisten sobre la evaluación de la relación riesgos/beneficios como criterio de protección del participante, y la aplicación de éstos dependerá de los conocimientos y valores de los miembros del CBI. La evocación de un «riesgo razonable» dependerá de varios factores; entre ellos, de la enfermedad base, del contexto de la investigación y en último término del medicamento en estudio. Estamos de acuerdo con Rudra y Lenk (25) en que la evaluación de los riesgos debe ser progresiva y permanente desde el diseño de la investigación para establecer medidas de mitigación, y en que es necesario poner un límite absoluto de riesgos, en particular en pacientes extremadamente vulnerables. Un aspecto importante es el posible efecto psicológico de los medicamentos sujetos a investigación sobre los participantes. Si estos efectos secundarios no han sido previstos, es difícil que los propios participantes los asocien con el medicamento de estudio (16). ${ }^{36}$

Por otra parte, las reglas anteriores sobre la renovación de los miembros del CBI cada cuatro años representaban un hándicap para la acumulación de experiencia en el manejo de los protocolos clínicos y la inclusión de nuevos miembros. ${ }^{37}$ La falta de interés por la bioética hasta los dos años recientes ha significado la permanencia en algunos CBI de miembros que tienen funciones administrativas en su propia institución. Por ello, algunos miembros permanecen 
Principios y regulaciones que orientan la evaluación de ensayos clínicos...

en comités durante varios años y sólo ahora pueden ser reemplazados progresivamente, debido a un mayor interés por la bioética y a la formación de nuevos miembros. ${ }^{38}$ Para resolver esta causal de conflicto de interés, recientemente un CBI decidió no revisar los protocolos en los cuales participan los directivos de la institución. El CNBI y la sociedad en general deben abordar estos conflictos de intereses para proponer soluciones éticas a favor de una mejor calidad del trabajo de los CBI institucionales (26). Los programas de educación permanente de los miembros de los CBI, y particularmente los encuentros de los CBI y los estudios de casos, representan herramientas para mejorar la calidad de su trabajo (27). En ningún caso estos problemas pueden ser invocados a favor del desarrollo de comités «independientes» fuera de las instituciones, cuyas relaciones financieras con la industria farmacéutica representan conflictos de interés (28).

La participación de miembros externos es importante para la transparencia de los debates y la protección de los participantes. Los CBI actuales han integrado a personas externas a la institución, con diferentes resultados, debido al carácter honorífico de los miembros y a la imagen elitista de la ciencia y de los científicos. Los CBI encuestados cuentan todos con miembros externos, pero su capacidad de intervención en las discusiones sobre la calidad metodológica y ética de las investigaciones de medicamentos es muy dispar, a pesar de que participan en las capacitaciones. ${ }^{39} \mathrm{La}$ Ley 84, de mayo del 2019, exige que los CBI cuenten con un mínimo de $20 \%$ de miembros externos, y prevé que serán compensados por los gastos ocurridos para asistir a las reuniones y capacitaciones. ¿Cuál debe ser el perfil de estos miembros externos para reforzar la protección de los participantes frente a los posibles intereses institucionales?

Los informes mensuales de los CBI permiten registrar las investigaciones en salud que se desarrollan en el país, reflejando la voluntad de transparencia de los CBI y constituyendo una fuente de 
información importante para los investigadores, los decisores de salud y el público en general, para no duplicar investigaciones y estar alerta a las investigaciones no aprobadas. ${ }^{40}$ Pero no permiten evaluar la calidad de la investigación ni la protección de los participantes, de los investigadores y de las instituciones. Todavía falta abordar estos problemas complejos sobre la completa seguridad en las investigaciones con fármacos.

Las normas administrativas y financieras del sector público tienen por objetivo presentar una imagen de transparencia y responsabilidad. Sin embargo, constituyen un hándicap para la continuidad de las actividades previamente programadas; en particular para las actividades educativas, y representan un serio obstáculo para la supervisión continua de las investigaciones en curso. Esta ausencia de supervisión es el problema más serio percibido por los CBI, ya que las dificultades internas pueden ser resueltas progresivamente. ${ }^{41}$ Uno de los problemas éticos de la situación actual se debe a que los CBI no tienen otra alternativa más que confiar en el sentido ético del investigador y de su equipo para el desarrollo de la investigación. Por ello, los CBI deben insistir sobre la educación ética de los investigadores y en el desarrollo de un clima de confianza mutua, como base del trabajo conjunto para proteger a los participantes y sin entorpecer la investigación. A su vez, el establecimiento de normas claras de obligatorio cumplimiento por parte de la industria farmacéutica implica que los CBI estén en capacidad de hacerlas respetar.

La esperanza de un tratamiento, aun cuando todavía esté bajo investigación, constituye en sí mismo un incentivo para participar en él, cuando todavía no hay tratamiento basado en la evidencia. Esta situación representa un dilema bioético entre beneficencia (de un tratamiento posiblemente eficaz) y no maleficencia (no someter a los participantes a riesgos desconocidos), que los CBI resuelven según su sensibilidad. ¿La ética de la responsabilidad individual y social es una utopía? 
Principios y regulaciones que orientan la evaluación de ensayos clínicos...

\section{Conclusiones}

La reglamentación de las investigaciones clínicas en Panamá ha progresado a favor de los participantes, tal como se refleja en las leyes y normativas nacionales adoptadas desde 2003. Sin embargo, se han encontrado dos problemas no resueltos:

1. La garantía de los beneficios referidos a la salud y a la protección contra las situaciones adversas que dependen, además de la vigilancia ética de los CBI, de las políticas públicas en salud e investigación. El peso de las empresas nacionales e internacionales en las relaciones comerciales dejan pensar que la resolución de este problema será lenta. En este sentido, es importante el rol de los CBI, academias e investigadores en la educación de las asociaciones de pacientes y del público general sobre sus derechos.

2. La burocratización de los CBI y la transformación de los debates sobre la ética de la investigación en debates sobre el cumplimiento de las leyes y normas existentes en esta área. Los CBI deben incorporar distintas disciplinas y pensamientos plurales con el propósito de fomentar la deliberación acerca de las implicaciones de las investigaciones financiadas por la industria farmacéutica en el contexto panameño de desigualdades sociales extremas, y de las medidas de contención de este riesgo. La multiplicación de CBI y el tiempo necesario para adquirir la experiencia y la fortaleza moral de solicitar la corrección de algunos protocolos o de rechazarlos, representan una amenaza a la protección efectiva de los participantes. En este sentido merece la pena analizar la posibilidad de segregar Comités de Bioética de la Investigación con Medicamentos, especializando esas funciones como en el ejemplo español (Real Decreto 1090/2015, del 4 de diciembre (29).

El respeto a la vida y a los derechos humanos; a la salud, a la seguridad y a la igualdad, así como la protección de las personas y grupos vulnerables (Art. 3 y 15 de la DUDH); la responsabilidad social y la salud (Art. 14); el aprovechamiento compartido de los beneficios (Art. 15), así como sobre las prácticas transnacionales éticas 
(Art. 21) afirmados en la DUBDH, y los principios éticos de autonomía, beneficencia/no maleficencia y justicia, deben constituir los referentes éticos para la discusión y la búsqueda de soluciones a estos problemas.

\section{Agradecimiento}

A Salud y Fármacos y a la Universidad de El Bosque, Colombia, por la coordinación multinacional y por auspiciar las reuniones de coordinación iniciales.

\section{Notas bibliográficas}

${ }^{1}$ Chile se retiró y Costa Rica y El Salvador se incorporaron.

${ }^{2}$ Para ser consecuentes con la definición de las leyes panameñas sobre este tema, emplearemos el término de Comités de Bioética de Investigación (CBI), en lugar de CEI, ya que es el nombre oficial de estos comités en Panamá.

${ }^{3}$ Mientras se preparaba el artículo para su publicación, la Asamblea Nacional aprobó la ley 84 de investigación en 2019.

${ }^{4}$ Uno de ellos no fue incluido en el análisis por la poca información pública disponible.

${ }^{5}$ Los coordinadores realizaron entrevistas en profundidad a seis miembros de $\mathrm{CBI}$ que habían participado en la primera etapa elegidos al azar y a un nuevo miembro. El objetivo era aclarar dudas y testar si algunas propuestas que se estaban discutiendo en otros países serían aceptables en Panamá.

${ }^{6}$ Los miembros de la comunidad deben participar en un curso de ética de investigación y buenas prácticas clínicas en los primeros seis meses de su ingreso a un CBI.

${ }^{7}$ El CBI-ICGES tiene, además, dos secretarías administrativas, debido al volumen de protocolos sometidos a revisión.

${ }^{8}$ Hace algunos años, hubo una tentativa de presión que encontró una clara oposición. Según los miembros de los CBI entrevistados, no se ha vuelto a repetir desde los últimos cinco años.

${ }^{9}$ El mismo modifica el Decreto Ejecutivo 1843, del 16 de diciembre de 2014, que reglamentaba el CNBI de Panamá (GO 27,716), y hacía obligatorio el registro de todas las investigaciones en salud.

${ }^{10}$ En total, para los cuatro comités estudiados se aprobaron 165 protocolos desde 2007 hasta 2018, según información de sus sitios web. 
Principios y regulaciones que orientan la evaluación de ensayos clínicos...

${ }^{11}$ Los estudios anti-VIH son prolongaciones de estudios anteriores que permiten a los participantes recibirlos; el MINSA asegura el tratamiento del VIH-SIDA a la población pediátrica y a los adultos voluntarios. Sin embargo, existe la observación empírica de que el grupo adulto es más cumplido en el marco de un protocolo de investigación que en las clínicas de atención correspondientes; es una observación que merece un estudio psico-social que no ha sido realizado.

12 Se excluyeron los ensayos con vacunas, porque no se consideran medicamentos.

${ }^{13}$ Algunos comités exigen que estos datos sean compartidos con el Ministerio de Salud.

${ }_{14}$ Uno de los comités solicitó cambios importantes en un protocolo sobre la base de los datos encontrados, lo cual terminó con el retiro del proyecto de investigación de Panamá.

${ }^{15}$ No existe reglamentación sobre este punto y queda a criterio de cada CBI.

${ }^{16}$ Los investigadores deben informar quiénes cumplirán esta función; no pueden ser residentes o internos.

${ }_{17}$ Se deben destacar las investigaciones en oncología, que abarcan personas de los sectores público y privado.

${ }^{18}$ Es necesario investigar si los problemas de acceso a los servicios de salud influyen sobre la participación en las investigaciones clínicas.

${ }^{19}$ No habiendo ley que permita a los CBI solicitar esta información que es considerada confidencial por los investigadores y las instituciones, no es posible confirmar estos datos.

${ }^{20}$ Las investigaciones con placebo sin medicamento alguno han sido pocas: para virus sincitial respiratorio o vacunas nuevas, y los CBI exigen que el/la participante o su tutor esté informado de esta posibilidad.

${ }^{21}$ Las investigaciones con medicamentos posiblemente teratógenos incluyen la oferta de anticonceptivos probados y el seguimiento del embarazo deseado hasta el nacimiento.

${ }^{22}$ Las instituciones financiadores y los investigadores se han apoyado en la ausencia del marco legal para no contestar a los CBI que han solicitado esta información, y los propios CBI no han insistido.

${ }^{23}$ Los CBI deben asegurar que se cumplan las normas: envío de la información en los tiempos establecidos a la Dirección General del Ministerio de Salud y a las empresas farmacéuticas; informe de causalidad entre el medicamento investigado y el evento adverso. El reporte de los eventos adversos y de las desviaciones deben especificar las medidas que se tomaron para responder a ello.

${ }^{24}$ Estas tarifas no están sujetas a la revisión y control del Ministerio de Salud.

${ }^{25}$ La Ley 84 de 2019 abre una posibilidad, al exigir un contrato previo entre el investigador principal, la institución auspiciadora y la entidad financiera patrocinadora para desarrollar una investigación. Poco a poco el marco legal reemplaza a las dudas éticas.

${ }^{26}$ La revista Martes Financiero, que expone la visión del sector en Panamá, ha insistido sobre estas ventajas. Hernández K. 2020. [Consultado 8 de enero de 2020]. 
Disponible en: https://www.martesfinanciero.com/portada/multinacional-britanicacomprueba-ventajas-del-hub-farmaceutico/

${ }^{27}$ Inclusión del tratamiento antirretroviral para las mujeres embarazadas y de las vacunas contra neumococo, rotavirus, papilomavirus, en el Programa Nacional de Vacunación.

${ }^{28}$ Se desconocen los posibles conflictos de interés de estos grupos.

${ }^{29}$ Este acompañamiento se encuentra en el POE de acreditación del CNBI, y se espera que las discusiones para la reglamentación de la Ley 84 sobre investigación permitirán abordar varias de las inquietudes presentadas en este trabajo.

${ }^{30}$ Tampoco presentan el presupuesto local detallado, porque no existe una reglamentación al respecto.

${ }^{31}$ Los informes internacionales sobre el tema son objeto de mucha discusión en los medios de comunicación de Panamá. La Estrella de Panamá; 23 de enero de 2020. Disponible en: https://www.laestrella.com.pa/nacional/200123/panama-ocupa-posicion-numero-101-180-paises-indice-corrupcion

${ }^{32}$ Los miembros de los $\mathrm{CBI}$ encuestados no opinaron sobre este tema y no se encontró un POE específico. La opinión general es que es función de las autoridades administrativas nacionales e institucionales.

${ }^{33}$ Artículo 46: Toda propuesta de investigación internacional que se realice en el territorio nacional deberá contar con la contraparte responsable en el país y con el apoyo y respaldo debidamente formalizado. Previo al inicio del proyecto de investigación, se debe establecer el acuerdo respectivo entre las fuentes de financiación, la institución de la información y el investigador principal, para la gestión de los datos que puedan ser de importancia para la toma de decisión en salud pública, los aspectos económicos y demás derechos y obligaciones de las partes.

${ }^{34}$ La Ley 81 sobre Protección de Datos Personales (2019) fija el marco legal para la recopilación, manejo y conservación de los datos personales en la era del Big Data, y su aplicación comenzará en el 2021. Por su importancia, los CBI necesitarán capacitación para aplicarla a los ensayos clínicos.

${ }^{35}$ Todos los CBI encuestados han solicitado el apoyo de un experto externo sobre un tópico poco común con buena respuesta.

${ }^{36}$ Tanto el diseño como la duración de los ensayos clínicos (generalmente entre seis meses y un año) dificultan la observación de estos cambios. Sólo las investigaciones en oncología prevén un periodo de seguimiento de por lo menos cinco años.

${ }^{37}$ La Ley 84 intenta responder a esta situación con el aumento del periodo de participación de los miembros a seis años, renovables una sola vez.

38 Según Carla Sáenz, asesora de OPS en Bioética, esta permanencia no representa un problema, ya que se aporta una experiencia acumulada (entrevista por Skype con el CNBI).

${ }^{39}$ La entrevista de estos miembros externos no estaba prevista en el diseño de este trabajo, y queda como una tarea pendiente.

${ }^{40}$ Registro de protocolos, versión web Minsa disponible en: https://cnbi.senacyt.gob.pa/comites-acreditados/ e Informes de Comités de Bioéticas acreditados dis- 
Principios y regulaciones que orientan la evaluación de ensayos clínicos...

ponibles en: https://cnbi.senacyt.gob.pa/informes-de-comites-de-bioetica-institucionales/ [Consultado el 23 de julio de 2020]. Ambos son de acceso público.

${ }^{41}$ Es necesario que los CBI desarrollen experiencia para la protección de los participantes, detectando las posibles alteraciones, falsificaciones y omisiones de datos (particularmente de los eventos adversos) durante las auditorías propias.

\section{Referencias bibliográficas}

1. Universidad El Bosque, Departamento de Bioética, Cátedra Abierta de Investigación en Salud y Bioética. Panel sobre «La situación de los Comités de Ética de Investigación en América Latina y El Caribe», Auditorio Fundadores. Memoria de actividades realizadas. 12 de octubre de 2016; 29-32. [Consultado el 28 de diciembre de 2019]. Disponible en: https://issuu.com/catedraabiertadebioetica/docs/ memo ria_c_tedra_bio_tica_2016_15 https://doi.org/10.18359/rlbi.502

2. National Institutes of Health $(\mathrm{NIH})$. Aprenda más sobre la investigación clínica de los NIH. Investigación clínica. 2019. [Consultado el 25 marzo de 2020]. Disponible en: https://salud.nih.gov/investigacion-clinica/ https://doi.org/10.1016/s02149168(01)78771-2

3. Lolas F, Quezada A, Rodríguez E. Investigación en Salud. Dimensión Ética. CIEB, Universidad de Chile, Primera edición, marzo de 2006. [Consultado el 28 de diciembre de 2019]. 2006; 59-90. Disponible en: https://www.academia.edu/3581 6399/Investigacion_en_salud_dimension_etica_pdf https://doi.org/10.4067/s1726$569 \times 2006000200015$

4. Castro G. Panama, its politics, and the canal against itself. Oxford Research Encyclopedia of Politics. 2019 October 30. Disponible en https://oxfordre.com/politics/view/10.1093/acrefore/9780190228637.001.0001/acrefore-9780190228637-e 1705? rskey=FQGwVN\&result=320 https://doi.org/10.1093/acrefore/9780190228637.013.1705

5. Vergès C. Chapter 15, the history of bioethics in Panama. En Pessini L. et al. (eds). Ibero-American Bioethics: History and Perspectives. Philosophy and Medicine 106. Springer Science + Business Media. 2010; 175-192. Disponible en https:// books.google.com.pa/books?id=8TwP6Isp2eUC\&pg=PA175\&lpg=PA175\&dq= Verg\%C3\%A8s+C.+The+History+of+Bioethics+in+Panama\&source=bl\&ots=eJHu R1HWml\&sig=ACfU3U0UotPbKuhlelpNZ79LzQ71wQ7Lbw\&hl=es\&sa=X\&ved=2ah UKEwjd8cidnrLsAhWkpFkKHWljAzMQ6AEwB3oECAkQAg\#v=onepage\&q=Verg \%C3\%A8s\%20C.\%20The\%20History\%20of\%20Bioethics\%20in\%20Panama\&f =false https://doi.org/10.1007/978-1-4020-9350-0_15

6. Organización Mundial de la Salud. Guías operacionales para comités de ética que evalúan investigación biomédica. Product research and development team. $T D R / P R D / E T H I C S / 2000$. [Consultado el 6 de diciembre de 2019]. 2000; 1. Disponible 
en: https://apps.who.int/iris/bitstream/handle/10665/66641/TDR_PRD_ETHICS_2000.1_spa.pdf

7. Hospital del Niño, Dr. José Renán Esquivel (HDNDJRE). Reglamento del CBI. 2013. [Consultado el 2 de enero de 2020]. Disponible en: https://hn.sld.pa/wp-content/uploads/2018/02/ULTIMO-REGLAMENTO-INTERNO-DEL-CE-JULIO-2013. pdf https://doi.org/10.37980/im.journal.rspp.20191565

8. Instituto Conmemorativo Gorgas de Estudios en Salud, CBI. [Consultado el 2 de enero de 2020]. Disponible en: http://www.gorgas.gob.pa/cbi-inicio/

9. Comité Nacional de Bioética de Investigación. Panamá. [Consultado 2 de febrero de 2020]. Disponible en: https://cnbi.senacyt.gob.pa

10. Pacífica Salud. Comité de Bioética. [Consultado el 23 de julio de 2020]. Disponible en: https://www.pacificasalud.com/single/es/comite-de-bioetica-en-investigacion

11. UNESCO. Guía NN 1. Creación de Comités de Bioética. UNESDOC, Biblioteca Digital. 2005. [Consultado el 20 de diciembre de 2019]. Disponible en: https://unesdoc.unesco.org/ark:/48223/pf0000139309_spa

12. UNESCO. Guía No 2. Funcionamiento de los comités de bioética: procedimientos y políticas. UNESDOC Biblioteca Digital. 2006. [Consultado el 20 de diciembre de 2019]. Disponible en: https://unesdoc.unesco.org/ark:/48223/pf00001473 92_spa

13. Fuentes $D$, Revilla $D$. Acreditación de Comités de Ética en Investigación, como parte de un proceso. An Fac Med Lima. [Consultado el 6 de julio de 2017]. 2007; 68(1): 67-74. Disponible en: https://www.redalyc.org/pdf/379/37968109.pdf https://doi.org/10.15381/anales.v68i1.1241

14. Ministerio de Salud (MINSA). Ley 84 del 14 de mayo de 2019: Que promueve la investigación para la salud y establece su rectoría y gobernanza, y dicta otras disposiciones. Gaceta Oficial Digital No. 28775-A. 2019. [Consultado el 21 de enero de 2020]. Disponible en: http://www.minsa.gob.pa/sites/default/files/general/ley_ 84_de_14_de_mayo_de_2019_regulaips.pdf https://doi.org/10.26439/ulima.tesis/ 10065

15. Minaya GE, Fuentes-Delgado DJ, Ugalde A, Homedes N. A missing piece in clinical trial inspections in Latin America: Interviews with research subjects in Peru. J Empir Res Hum Res Ethics. [Consultado el 21 de enero de 2020]. 2017; 12(4), 232-245. https://doi.org/10.1177/1556264617720756

16. Farmaindustria. Ensayos clínicos, claves para desarrollar nuevos fármacos y valiosos para la economía y el ahorro de costes sanitarios. Noticias. España. 16 de mayo de 2019. [Consultado el 15 de octubre de 2019]. Disponible en: http:// www.pmfarma.es/noticias/27172-ensayos-clinicos-claves-para-desarrollar-nuevos-farmacos-y-valiosos-para-la-economia-y-el-ahorro-de-costes-sanitarios.html https://doi.org/10.18567/sebbmdiv_rpc.2015.10.1

17. Angel M. Transparency hasn't stopped drug companies from corrupting medical research. New York Times. 2018; September 14. Disponible en: https:// www.nytimes.com/2018/09/14/opinion/jose-baselga-research-disclosure-bias.html 
Principios y regulaciones que orientan la evaluación de ensayos clínicos...

18. Lorenzo C, Garrafa V. Ensayos clínicos, Estado y sociedad: ¿dónde termina la ciencia y empieza el negocio? [Debate]. Salud Colectiva. 2011; 7(2):166-170. https://doi.org/10.18294/sc.2011.376

19. Bachelet VC, Pardo-Hernández $\mathrm{H}$. Calidad del reporte y riesgo de sesgo de los ensayos clínicos aleatorizados. Medwave. [Consultado el 2 de febrero de 2020]. 2019; 19(1): e7573. Disponible en: https://www.medwave.cl/link.cgi/Medwave/Perspectivas/Editorial/7573 https://doi.org/10.5867/medwave.2019.01.7573

20. Abbott L, Grady C. A systematic review of the empirical literature evaluating IRBs: What we know and what we still need to learn. J. Empir. Res. Hum. Res. Ética. $2011 ; 6(1)$ : 3-19. https://doi.org/10.1525/jer.2011.6.1.3

21. Rodwin MA. Institutional corruption and the pharmaceutical policy introduction. $J$ Law Med Ethics. 2013; 41(3): 544-552. https://doi.org/10.1111/jlme.12062

22. Weissman JS, Koski G, Vogeli C, Thiessen C, Campbell EG. Opinions of IRB Members and chairs regarding investigators' relationships with industry. $J$ Empir Res Hum Res Ethics. 2008; 3(1): 3-13. https://doi.org/10.1525/jer.2008.3.1.3

23. Reuben SH, Milliken EL, Paradis LJ. The future of cancer research: Accelerating scientific innovation. President's cancer panel Annual Report 2010-2011. National Institutes of Health National Cancer Institute. 2012. [Consultado el 21 de agosto de 2019]. Disponible en: https://deainfo.nci.nih.gov/Advisory/pcp/annualReports/pcp10-11rpt/FullReport.pdf

24. Redacción Médica. Sesgo en protocolos de investigación oncológica. 19 de septiembre de 2019. [Consultado el 18 de octubre de 2019]. https://www.redaccionmedica.com/secciones/oncologia-medica/el-50-de-ensayos-para-la-aprobacion-de-oncologicos-plantea-dudas-de-sesgo-9940

25. Rudra P, Lenk C. Process of risk assessment by research ethics committees: foundations, shortcomings and open questions. J Med Ethics. 2020 Jan 10: medethics-2019-105595. https://doi.org/10.1136/medethics-2019-105595

26. Klitzman R. Views and Experiences of IRBS concerning research integrity. J Law Med Ethics. 2011; 39(3): 513-528. https://doi.org/10.1111/j.1748-720x.2011.00618.x

27. Ong YT, Yoon NYS, Yap HW, et al. Training clinical ethics committee members between 1992 and 2017: Systematic scoping review. J Med Ethics. [Consultado el 17 de abril de 2020]. 2020; 46: 36-42. Disponible en: https://jme.bmj.com/content/ 46/1/36 https://doi.org/10.1136/medethics-2019-105666

28. Kaplan S. In clinical trials, for-profit review boards are taking over for hospitals. Should they? STAT. 2016. [Consultado el 2 de enero de 2020]. Disponible en: https://www.statnews.com/2016/07/06/institutional-review-boards-commercial-irbs/ 29. Molina ME, Arroba BML. Nueva regulación de ensayos clínicos con medicamentos en España. Pediatría de Atención Primaria. [Consultado el 18 de marzo de 2018]. 2016; 18: e229-e237. Disponible en: https://pap.es/articulo/12440/nueva-regulacion-de-ensayos-clinicos-con-medicamentos-en-espana https://doi.org/ 10.1016/s0300-2896(03)75385-x

Medicina y Ética - Abril-Junio 2021 - Vol. 32 - Núm. 2 
